\title{
Drossbach, Gisela, Von der Ordnung zur Norm : Statuten in Mittelalter und Früher Neuzeit
}

\section{Rainer Maria Kiesow}

\section{OpenEdition}

\section{Journals}

Édition électronique

URL : http://journals.openedition.org/ifha/6720

DOI : $10.4000 /$ ifha. 6720

ISSN : 2198-8943

Éditeur

IFRA - Institut franco-allemand (sciences historiques et sociales)

Référence électronique

Rainer Maria Kiesow, «Drossbach, Gisela, Von der Ordnung zur Norm : Statuten in Mittelalter und Früher Neuzeit », Revue de l'IFHA [En ligne], Date de recension, mis en ligne le 01 janvier 2012, consulté le 22 septembre 2020. URL : http://journals.openedition.org/ifha/6720 ; DOI : https://doi.org/10.4000/ifha. 6720

Ce document a été généré automatiquement le 22 septembre 2020.

(C)IFHA 


\title{
Drossbach, Gisela, Von der Ordnung zur Norm : Statuten in Mittelalter und Früher Neuzeit
}

\author{
Rainer Maria Kiesow
}

1 Pour être clair d'emblée : « De l'ordre à la norme : les statuts au Moyen-âge et dans les temps modernes » est une œuvre collective d'une grande érudition. Celui qui voudra s'informer de ce qu'on sait aujourd'hui sur les règles, statuts, coutumiers de l'abbaye de Saint-Ruf dans le Sud de la France (U. Vones-Liebenstein), sur les institutions ecclésiastiques et en particulier les statuts des diocèses en Prusse (A. Mentzel-Reuters), sur les synodes et les nouveaux médias du XVe siècle (H. J. Mierau), sur les règlements internes de la papauté (A. Meyer, T. Schmidt), sur les ordres/statuts dans des régions et villages, que ce soit en Angleterre, en Allemagne ou en Sardaigne (M. Kaufhold, K. Härter, C. Birr, N. Pes), sur les institutions statutaires, normatives, législatives des villes italiennes et allemandes, de Paris et Bologne, dans les communautés juives (R. Gibbs, M. Ascheri, F. Schmieder, H.-G. Hermann, W. J. Courtenay, S. Litt), sur les statuts et cérémonies dans l'aristocratie (J. Sarnowsky, G. Kerscher), sur les communautés de frères en Angleterre, Italie, France et Allemagne (K. Behrens, A. Esposito, T. Frank, L. Schneider, K. Gvozdeva) - donc celui qui voudra jouir d'un vaste panorama de la situation des statuts et des normes de ces quelques siècles avant le XVIIe/XVIIIe trouvera largement de quoi satisfaire sa curiosité. Car c'est une longue et étrange période que celle qui ne connaissait pas encore LA loi, donc pas encore de norme unique, cet échafaudage moderne d'une normativité dépliée en statuts, règlements, normes qui dans toutes leurs différenciations découlent pourtant d'une seule source, la loi, la constitution, la législation conforme à celle-ci, même dans les plus petites particules normatives.

2 Le titre "De l'ordre à la norme » suggère une sorte de préhistoire de cette modernité " légale » dans laquelle nous vivons (en Europe). Ce qu'on trouve après lecture : un énorme fatras. 
3 Un fatras de mots divers, de concepts contradictoires, d'images superposées. La directrice de la publication, G.D. le dit elle-même : " On ne cherche pas une définition commune des statuts » (p. 369). Reste donc à dire que les statuts sont des réglementations locales, plutôt internes et descriptives. Et un ordre communautaire se donnant de telles règles tend vers un régime normatif : donc d'abord l'ordre, et après la norme. Ainsi pourrait-on décrire le statut des statuts de la période concernée.

4 Reste le fatras, car, indépendamment de la modestie théorique de G.D., certains auteurs, notamment F. Schmieder, contestent d'emblée l'utilisation de la notion de " statut » puisque celle-ci rend les textes et pratiques des communautés en question plus obscurs qu'elle ne les clarifie, vu la diversité des sociétés et de leurs systèmes de droit. Ce qui provoque, bien sûr, des répliques, par exemple de la part d'historiens du droit comme H.-G. Hermann qui insiste sur l'aspect « normatif » des statuts pouvant justement dessiner un droit autonome - des villes par exemple.

5 Le fatras néanmoins est de règle : diversité géographique et temporelle, multitude d'objets (église, ville, monastère, régions, village, pape etc.). Les statuts, comme expression des sources, autant que comme concept de la recherche d'aujourd'hui, se trouvent partout. S'y ajoutent les horizons divers des chercheurs des statuts, à savoir des historiens du droit, de l'art, de la philosophie, des historiens tout court, des musicologues ou encore des philologues (malheureusement il n'y a aucune indication du « métier » des auteurs, il faut les connaître ou deviner). Dès lors, la recherche d'une définition commune du « statut » dans ce volume semble complètement vaine.

On se demande finalement pourquoi ce titre un peu pompeux « De l'ordre à la norme ». Les textes sont très spécialisés et chacun reste cantonné à son domaine et a tout l'air d'une monade. Ce sont des enfants uniques des auteurs qui entre eux ont peu à faire, sauf que le mot «statut » apparaît constamment. Et si déjà la directrice ne voit pas de cohérence («On ne cherche pas ... ») - comment le lecteur oserait-il en chercher une ? « Haec sunt statuta » (G.D., p. 369) - en effet, le pluriel domine, une réflexion, sur ce que « norme ", « statut », « ordre " pourraient signifier, manque.

7 Le fatras, certes, est précédé par une sorte d'introduction, intitulée « Le charme de la multitude ", mais Cl. Märtl n'y consacre qu'une page et demie. Suivent, comme « prologue ", deux autres textes introductifs de deux célébrités en histoire du droit (canonique) : P. Landau et K. Pennington. Landau consacre déjà le double, soit 3 pages, à une réflexion sur les concepts de norme, ordre, statut et coutume. Mais l'ensemble reste trop sommaire et trop vague et le " peut-être " y règne en maître. Pourquoi pas? C'est honnête, mais il faudrait plus, surtout plus d'acharnement théorique pour valider une phrase comme : «Le XXIIe siècle est le siècle juridique par excellence auquel nous devons la redécouverte de la législation dans la culture juridique européenne » (p. 15). Pennington, quant à lui, s'efforce, dans sa contribution qu'on peut appeler " article ", de montrer comment le droit romain "retrouvé "s'est frayé son chemin jusqu'aux juristes « non-romains » et aux communautés locales, comment donc il est devenu une " science pratique » (p. 17). Très bien, peut-être aussi juste, mais pour la " preuve », il faudrait se lancer dans cette pratique même, une entreprise énorme, européenne, une recherche du droit de tous les jours dans les localités du Portugal jusqu'à Byzance, une Byzance d'ailleurs qui manque systématiquement dans ce genre d'étude. Chacun son métier, Pennington en reste ainsi à une histoire " intellectuelle ", " savante ", principalement une histoire de la relation entre droit romain et droit canon. Ce qui l'intrigue, ce sont les empreintes du droit romain sur « les coutumes, les institutions, le 
droit canon et la vie intellectuelle de la société médiévale » (p. 38). De l'ordre à la norme, de l'ordre aux innombrables statuts dans le marais des pratiques plus ou moins juridiques en Europe - ce chemin ne peut pas être emprunté avec une vision « top down » à la Pennington.

Le fatras de ces difficultés de base qu'on rencontre dans ce volume n'est donc point mis en ordre par le prologue, ce qui serait (« On ne cherche pas ... ») certainement aussi trop demander. Mais pour un livre qui s'intitule, je me répète, « De l'ordre à la norme », une réflexion sur l'ordre des normes s'impose. Une histoire qui ne s'occupe que peu du façonnement de ces notions-clefs, trop banalement attribuées aux « sources ", ne sait plus quoi dire et ne dit finalement pas grand' chose.

Reste l'érudition. C'est beaucoup. Je ne crois pas que cela suffise pour comprendre quoi que ce soit, par exemple la constitution juridique d'une société vivante ou morte. Et il y a des opérations qui montrent que toute érudition est susceptible d'érosion. A. Meyer, dans son article exceptionnellement précis et érudit sur les règles de la chancellerie papale au Moyen Âge tardif, se confronte aux textes, aux règles manuscrites de diverses mains et divers temps. Il présume, en passant, que « la version plus longue ou plus prolixe d'une règle est la plus ancienne " (p. 102). C'est tout un séminaire de méthodologie, théorie, histoire du droit qu'on pourrait concevoir à partir de cette présomption. Pour un juriste, ce serait et cela a été, tout le contraire, on le voit tous les jours. Le style du droit, législatif ou bureaucratique, se complique au cours du temps. Peut-être y a-t-il des raisons de penser qu'au bureau du Pape, il y a 650 ans, ce n'était pas ainsi. Mais on voudrait bien avoir une explication de ce fait qui en outre est crucial pour toute une chronologie "de l'ordre à la norme ». L'érudition n'échappe pas à l'érosion de ce genre de présomptions, dont chaque description historique regorge. " On ne cherche pas ... ». C'est humble, certes, mais c'est avant tout une capitulation pour préserver un champ de recherche sans théorie. Mais, même pour dater deux écritures manuscrites, il faut une réflexion théorique. Donc : cherchez! On n'aurait peut-être pas adhéré, mais au moins y aurait-il eu matière à s'accorder - ou pas. 